\title{
ВЛИЯНИЕ ИНФОРМАЦИОННОЙ СРЕДЫ НА СУБЪЕКТЫ МАЛОГО И СРЕДНЕГО ПРЕДПРИНИМАТЕЛЬСТВА
}

\author{
(c) 2020 Родионов Дмитрий Григорьевич \\ доктор экономических наук, профессор, Высшая инженерно-экономическая школа \\ Санкт-Петербургский политехнический университет Петра Великого (СПбПУ), \\ Россия, Санкт-Петербург \\ E-mail: dmitry.rodionov@spbstu.ru \\ (c) 2020 Ялымов Сергей Вячеславович \\ Высшая инженерно-экономическая школа \\ Санкт-Петербургский политехнический университет Петра Великого (СПбПУ), \\ Россия, Санкт-Петербург

\section{(c) 2020 Конников Евгений Александрович} \\ кандидат экономических наук, Высшая инженерно-экономическая школа \\ Санкт-Петербургский политехнический университет Петра Великого (СПбПУ), \\ Россия, Санкт-Петербург \\ E-mail: konnikov.evgeniy@gmail.com
}

Сектор малого и среднего предпринимательства является одним из системообразующих в рамках национальной экономики. Несмотря на относительно низкую долю ВВП, данный сектор решает крайне значимые социальные функции, ключевой из которых является формирование региональных рынков труда и обеспечение занятости социально-необеспеченных слоев населения. При этом сектор малого и среднего предпринимательства крайне волатильный и чувствителен к институциональным изменениям, в частности к изменениям в информационной среде. При этом, на данный момент не существует единой методологии оценки влияния информационной среды на представителей сектора малого и среднего бизнеса. В рамках данной статьи авторы предлагают структуру и элементы данной методологии, а также базовые инструменты квантификации данных элементов.

Ключевые слова: сектор малого и среднего предпринимательства, МСП, информационная среда, тональность информационной среды, токены, лексический анализ

Информационная среда является субсредой макросреды субъектов малого и среднего предпринимательства (далее МСП). Данная субсреда является наиболее вариативной и комплексной, так как она отражает содержание информационного фона и специфику моментного взаимодействия субъектов МСП с субъектами микроинституциональной среды (такими как поставщики, потребители, конкуренты и т.д.) в контексте данного информационного фона. Влияние данной среды на развитие субъектов МСК крайне значимо, так как:

1. Информационная среда наиболее направленно и значимо оказывает влияние на восприятие субъекта МСП со стороны основных потребителей, что в свою очередь определяет колебания спроса. В качестве примера можно привести экспоненциальный прирост спроса на лимоны и имбирь на ранних этапах пандемии
COVID-19. Данный прирост обусловлен появлением в информационной среде необеспеченного какими-либо фактами слуха относительно пользы данных продуктов для профилактики соответствующего заболевания. Также, в качестве примера можно привести экспоненциальный прирост спроса на бытовую технику и иностранную валюту в начале 2014 года. Данный прирост был обусловлен появлением в информационной среде устойчивого понимания наступления экономического кризиса, что спровоцировало у потребителей ожидание продолжительной рецессии экономики. Приведенные в качестве примера и иные подобные проявления непосредственно отражают влияние информационной среды на спрос.

2. Субъекты МСП являются наиболее чувствительными к колебаниям спроса, вызванным изменениями в информационной среде. Более 
того, так как субъекты МСП в первую очередь зависимы от оборачиваемости активов, функция, которой можно описать реакцию субъектов МСП на колебания спроса, имеет экспоненциальный характер. В качестве примера можно привести рынок общественного питания в конце мая 2020 года. Несмотря на отмену самоизоляции, потребители все еще не готовы посещать заведения общественного питания, в связи с изменениями паттернов потребления, обусловленных массированным воздействием со стороны информационной среды. В связи с данным фактом подавляющее число заведений общественного питания не могут выйти на необходимый уровень оборачиваемости даже после отметены самоизоляции.

3. Влияние со стороны информационной субсреды может значительно дифференцироваться в зависимости от субъекта МСП, что определяет необходимость раздельного анализа информационной среды как самого предприятия, так и его конкурентов и комплименторов. Информационная среда может оказывать крайне направленной воздействие. В качестве примера можно привести прецедент 2017 года с компанией Samsung и ее смартфоном Galaxy Note 10. Конструктивное несовершенство данного агрегата провоцировало непроизвольное возгорание аккумуляторных элементов. Несмотря на то, что данный конструктивный дефект был устранен в течение нескольких недель, вероятность возгорания была кране мала, а уже проданные смартфоны были отозваны и заменены на смартфоны с устраненным дефектом, данная новость была крайне быстро распространена в информационной среде, что в свою очередь привяло к сокращению объёмов продаж. Данный факт стал элементом популярной культуры, что в свою очередь даже на данный момент может оказывать влияние на спрос со стороны отдельных потребителей. При этом, спрос на товары компаний конкурентов значительно повысился в соответствующий период времени. Также, в качестве еще одного примера можно привести последствия новости об исключении из фондов компании Amazon фильма «Унесенные ветром», в связи с недопустимым контекстом демонстрации рабства. Следствием данного информационного посыла стал ажиотажный спрос на данный фильм, как на физических носителях, так и в электронном виде. В результате компания Amazon смогла в течение трех дней распродать значительную долю запасов физических носителей с данным произведением кинематографа.

Информационная среда может быть представлена совокупностью каналов распространения информации, а именно: радио, телевидение, печатные СМИ, интернет СМИ, социальные сети и т.д. Сама информация при этом подается в естественной форме, что значительно усложняет процесс ее анализа. Для целей анализа влияния со стороны информационной среды необходимо использовать либо экспертный подход, либо обрабатывать и квантифицировать естественную информацию. В рамках данной статьи предлагается модель агрегирования, обработки и квантификации естественной информации. Данный автоматизированный подход выбран с целью сокращения уровня субъективности результирующего показателя, что неизбежно при условии исключительно экспертного подхода.

Структура влияния информационной среды может быть дифференцирована с точки зрения двух базовых компонент:

1. Тональная компонента информационной среды. Тональность информационной среды - это эмоциональное отношение автора объекту информационного фона, отраженному в определённом информационном массиве. Объектами могут выступать события, процессы, продукты, бренды, непосредственно сами субъекты МСП и многое другое. Тональность всего текста в целом можно определить как функцию лексических тональностей составляющих его единиц и правил их сочетания [1]. Анализ тональности информационной среды сосредоточен на определении полярности эмоциональных оценок в исследуемом информационной фоне, который содержит мнения, суждения, эмоции, отношение к сущностям, личностям, вопросам, событиям, темам и их атрибутам, а также условную фактологию [2]. Сущностно, анализ данной компоненты информационной среды призван ответить на вопрос как социум (его ячейка, группа или отдельный субъект) относится к объекту исследования. В рамках наиболее упрощенной модели данное отношение может быть охарактеризовано как положительное, отрицательное или нейтральное.

2. Содержательная компонента информационной среды. Данная компонента характеризует лексическое наполнение информационного фона. Информационный фон может быть представлен (в рамках описания данной 
компоненты) как совокупность элементарных лексически-смысловых единиц, а именно слов и словосочетаний. Расщепив информационный фон на данные элементарные частицы, приведя их к базовой словарной форме и отчистив от вспомогательных элементов, обладающих низкой содержательной нагрузкой (союзов, предлогов, местоимений и т.д.) формируется условное облако токенов. Токен - элементарная содержательная единица информационного фона. Каждый из токенов, за счет своего наличия в информационном фоне, может оказывать воздействие на восприятие потребителями того или иного объекта, которыми могут быть как сами субъекты МСП, так и ТРУ субъектов МСП, их конкурентов и комплименторов. Анализ облака токенов позволит охарактеризовать вектор и силу воздействия информационной среды в первую очередь на процесс трансформации спроса со стороны групп потребителей.

Процесс цифровизации жизнедеятельности привел к фактическому удалению барьеров, направленных на ограничение доступа к информации. В связи с этим, влияние информационной среды на потребителей значительно усиливается с каждым годом. Для целей оценки приведенных компонент предлагается использовать автоматизированный анализ информационного фона посредствам языка программирования Python и специализированных библиотек. Источником информационного фона в свою очередь могут выступать новостные сайты и сообщества в социальных сетях, чья принадлежность определяется конкретным кейсом.

Рассмотрим в первую очередь тональную компоненту информационной среды. Как отмечалось выше, тональность, в упрощенной форме, может быть определена как позитивная, негативная и нейтральная. При этом тональность необходимо разделитель на следующие элементы: общая тональность и направленная тональность.

Общая тональность информационной среды. Данный параметр может определяться по результатам анализа новостного массива, не имеющего содержательной специфики. Данный параметр сущностно отражает тональные характеристики окружающей информационной среды, а следовательно влияние, которое может оказывать информационная среда на все элементы микросреды. Источником информации в данном случае могут выступать новостные сайты. Наиболее эффективным инструментом для определения тональности материалов, представленных на русском языке, является библиотека Dostoevsky. Данная библиотека позволяет определить такие тональные характеристики текста, как нейтральность, проходной характер, негативность, позитивность и естественность языка. Каждая из приведенных характеристик измеряется от 0 до 1. Для целей формируемой модели, наиболее значимыми параметрами является негативность и позитивность. При высоком уровне позитивности информационной среды потребители склонны увеличивать потребление, что неизбежно положительно сказывается на секторе МСП. В случае увеличения уровня негативности информационной среды, потребители, наоборот, склонны наращивать сбережения. В следствии вышесказанного, параметр общей тональности информационной среды может быть определен как отношение соответствующих параметров.

$$
T^{s}=\frac{t_{p}}{t_{n}}
$$

Где:

1. Ts - общая тональность информационной среды (измеряется в условных баллах);

2. $\mathrm{t}_{\mathrm{p}}$ - уровень позитивности информационной среды, определенный в соответствии с библиотекой Dostoevsky (измеряется в условных баллах от 0 до 1);

3. $t_{n}$ - уровень негативности информационной среды, определенный в соответствии с библиотекой Dostoevsky (измеряется в условных баллах от 0 до 1$)$.

Увеличение данного показателя неизбежно положительно сказывается на субъектах микросреды МСП. В тоже время, значительно большее влияние на субъекты МСП оказывает показатель направленной тональности. Данный показательно характеризует информационной фон, концентрирующийся вокруг конкретных токенов. В частности, если предприятие МСП занимается предоставлением услуг общественного питания, то в качестве ключевых токенов можно выделить: кафе, ресторан, общепит, еда, а также сочетания данных токенов и географические характеристики (название города и улицы, где расположено предприятие). Также, в качестве токенов в данном случаем могут выступать название самого предприятия, ФИО его руково- 
дителя или сотрудников и иная подобная информация. Соответствующий показатель может быть определен направленно по отношению ко всем выделенным ранее субъектам МСП:

$$
\begin{aligned}
& T^{v^{e}}=\frac{t_{p}{ }^{e}}{t_{n}{ }^{e}} \\
& T^{v^{c}}=\frac{t_{p}{ }^{c}}{t_{n}{ }^{c}} \\
& T^{v^{s}}=\frac{t_{p}{ }^{s}}{t_{n}{ }^{s}} \\
& T^{v^{c o m}}=\frac{t_{p}{ }^{c o m}}{t_{n}{ }^{c o m}}
\end{aligned}
$$

Где:

1. Tve - направленная тональность информационной среды субъекта МСП (измеряется в условных баллах);

2. Тvс - направленная тональность информационной среды конкурентов субъекта МСП (измеряется в условных баллах);

3. Tvs - направленная тональность информационной среды поставщиков субъекта МСП (измеряется в условных баллах);

4. Tvсот - направленная тональность информационной среды комплименторов субъекта МСП (измеряется в условных баллах);

5. $\mathrm{t}_{\mathrm{p}} \mathrm{e}-$ уровень позитивности информационной среды субъекта МСП, определенный в соответствии с библиотекой Dostoevsky (измеряется в условных баллах от 0 до 1);

6. $\mathrm{t}_{\mathrm{n}} \mathrm{e}$ - уровень негативности информационной среды субъекта МСП, определенный в соответствии с библиотекой Dostoevsky (измеряется в условных баллах от 0 до 1);

7. $t_{\mathrm{p}} \mathrm{c}$ - уровень позитивности информационной среды конкурентов субъекта МСП, определенный в соответствии с библиотекой Dostoevsky (измеряется в условных баллах от 0 до 1);

8. $t_{n} \mathrm{c}-$ уровень негативности информационной среды конкурентов субъекта МСП, определенный в соответствии с библиотекой Dostoevsky (измеряется в условных баллах от 0 до 1);
9. $\mathrm{t}_{\mathrm{p}} \mathrm{s}-$ уровень позитивности информационной среды поставщиков субъекта МСП, определенный в соответствии с библиотекой Dostoevsky (измеряется в условных баллах от 0 до 1);

10. $\mathrm{t}_{\mathrm{n}} \mathrm{s}$ - уровень негативности информационной среды поставщиков субъекта МСП, определенный в соответствии с библиотекой Dostoevsky (измеряется в условных баллах от 0 до 1);

11. $\mathrm{t}_{\mathrm{p}} \mathrm{com}-$ уровень позитивности информационной среды комплименторов субъекта МСП, определенный в соответствии с библиотекой Dostoevsky (измеряется в условных баллах от 0 до 1);

12. $t_{n}{ }^{c o m}-$ уровень негативности информационной среды комплименторов субъекта МСП, определенный в соответствии с библиотекой Dostoevsky (измеряется в условных баллах от 0 до 1).

Далее необходимо рассмотреть содержательную компоненту. Для каждого субъекта МСП может быть составлено собственное облако токенов, в соответствии с которыми производится фильтрация информационного фона, данный массив может быть назван «облако целевых токенов». В данном случае стоит выдвинуть гипотезу, согласно которой частота упоминаний целевых токенов, при учете динамики направленной тональности, положительно влияет на микросреду МСП. Таким образом, можно сформировать четыре направленных показателя, характеризующих данное влияние.

$$
\begin{aligned}
& N^{v^{e}}=\frac{T^{v^{e}}}{T^{v^{e}}} * \frac{n^{e}}{N} \\
& N^{v^{c}}=\frac{T^{v^{c}}}{T^{v^{c}}} * \frac{n^{c}}{N} \\
& N^{v^{s}}=\frac{T_{1}^{s}}{T^{v^{s}}} * \frac{n^{s}}{N}
\end{aligned}
$$

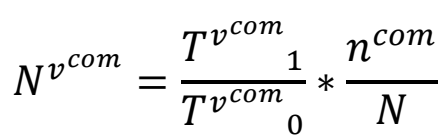


Где:

1. Nve - коэффициент целевого содержания информационной среды субъекта МСП (измеряется в условных баллах);

2. Nvc - коэффициент целевого содержания информационной среды конкурентов субъекта МСП (измеряется в условных баллах);

3. Nvs - коэффициент целевого содержания информационной среды поставщиков субъекта МСП (измеряется в условных баллах);

4. Nvcom - коэффициент целевого содержания информационной среды комплименторов субъекта МСП (измеряется в условных баллах);

5. nе - число упоминаний целевых токенов субъекта МСП в общей информационной среде (измеряется в единицах);

6. $\mathrm{n}^{\mathrm{c}}-$ число упоминаний целевых токенов конкурентов субъекта МСП в общей информационной среде (измеряется в единицах);

7. $\mathrm{ns}-$ число упоминаний целевых токенов поставщиков субъекта МСП в общей информационной среде (измеряется в единицах);

8. ncom - число упоминаний целевых токенов комплименторов субъекта МСП в общей ин- формационной среде (измеряется в единицах);

9. $\mathrm{N}$ - число токенов в общей информационной среде.

Как можно видеть данные показатели корректируются на изменение направленной тональности (индексы 1 и 0 обозначают отчетный и базисный период соответственно).

Таким образом, влияние информационной субсреды макросреды может быть выражено посредствам 9 описанных показателей. Необходимо в очередной раз подчеркнуть, что именно информационная среда оказывает наибольшее влияние на микросреду МСП, так как в условиях цифровой трансформации именно данная среда в первую очередь оказывает влияние на спрос, который в свою очередь является базисом существования сектора МСП в принципе. Данная конверсионная модель представлена на рисунке 1.

Проставленная модель является универсальной с отраслевой точки зрения. Дальнее развитие полученной модели подразумевает ее апробации и последующую разработку инструментов принятия решений на основе получаемых результатов.

\section{Факторы информационной среды}
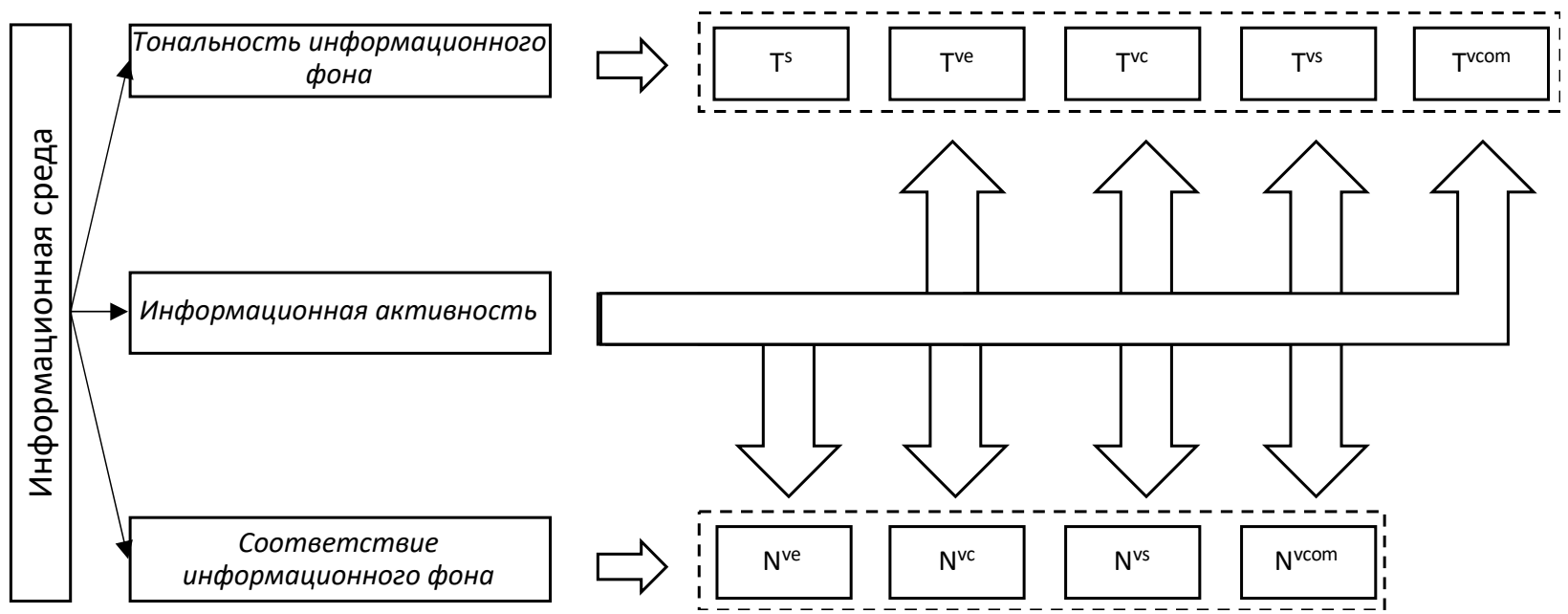

Puc. 1. Модель количественного выражения влияния факторов информационной среды 


\section{Библиографический список}

1. Bing Liu. Sentiment Analysis and Subjectivity // Handbook of Natural Language Processing (англ.) / под ред. N. Indurkhyа и F. J.Damerau. -2010.

2. Liu, B., \& Zhang, L. (2012). A Survey of Opinion Mining and Sentiment Analysis. In C. C. Aggarwal and C.X.Zhai (eds.), Mining Text Data, DOI 10.1007/978-1-4614-3223-4_13, @ Springer Science+Business Media, LLC

3. Негашев Д.С., Родионов Д.Г. Управление ликвидностью малого бизнеса // Российский экономический интернет-журнал. 2018. № 4. С. 80

4. Родионов Д.Г., Гильманов Д. В. Совершенствование моделей и механизмов управления малыми инновационными предприятиями // Экономика и предпринимательство. 2016. № 12-3 (77). С. 365-372

5. Трушникова И.О., Ширшова О.И., Погребова О.А. Количественные методы исследований в маркетинге // Санкт-Петербург, 2017

6. Юлдашева О.У., Трефилова И. Н., Погребова О.А. Методология разработки клиентоориентированных инновационных бизнес-моделей // Конкурентоспособность в глобальном мире: экономика, наука, технологии. 2017. № $8-4$ (55). С. $145-150$. 